\title{
One-Electron Spectral Functions of the Attractive Hubbard Model for Intermediate Coupling
}

\author{
Maxim Yu. Kagan ${ }^{(a)}$, Raymond Frésard ${ }^{(b)}$, Massimiliano Capezzali ${ }^{(b)}$ and Hans $\operatorname{Beck}^{(b)}$ \\ (a) P.L. Kapitza Institute for Physical Problems, Kosygin Str. 2, 117334 Moscow, Russian Federation \\ (b) Institut de Physique, Université de Neuchâtel, Rue A.L. Breguet 1, 2000 Neuchâtel, Switzerland
}

\begin{abstract}
We calculate the one-electron spectral function of the attractive (negative- $U$ ) Hubbard model. We work in the intermediate coupling and low density regime and obtain the self-energy in an approximate analytical form. The excitation spectrum is found to consist of three branches. The results are obtained in a framework, based on the self-consistent T-matrix approximation, which is compatible with the Mermin-Wagner theorem.
\end{abstract}

PACS numbers: 74.20Mn, 74.25.-q, 74.72.-h

\section{(A) INTRODUCTION}

The Hubbard model involving electrons on a lattice, subject to an attractive interaction when they are on the same site, is one of the simplest models for describing superconductivity. Despite of its simplicity, it has turned out to be very challenging for the theoreticians to give a simple description of its properties which is valid in the various regimes of coupling strength. In the weak coupling regime, the link with BCS theory of superconductivity has been done by Nozières and Schmitt-Rink [1]. At sufficiently low $T$, an instability of the Fermi sea towards superconductivity occurs. In three dimensions, the transition is essentially mean-field in character. In the opposite strong coupling limit $(|U| \rightarrow \infty)$, the electrons form bound pairs which are immobile since they can only move via virtual ionization with an infinite energy barrier. However, for large but finite $U$, those bound pairs essentially behave like heavy hard core bosons (with an effective mass $m^{*} \sim m \frac{U}{t}$ ) which are undergoing BoseEinstein condensation at sufficiently low $T$. On the lattice, $T_{C}$ vanishes in the limit $|U| \rightarrow \infty$, while in the continuum limit, it remains finite [2]. This difference is due to the absence of a pair hopping term when working on the lattice.

In the intermediate coupling regime, the physics will be dominated by the interplay between the quasi-particles and the bound pairs, which may lead to non-trivial behavior.

Even though it is still lacking a microscopical derivation, the model is interesting in its own right, since it allows for studying various routes leading to superconductivity. Since the interaction is local, it will be $s$ wave superconductivity, but the generalization to nonlocal interaction can be considered [3]. In the weak coupling regime, perturbation theory is expected to work, and this has been worked out by a series of authors [4], some of them focusing on 2-d systems [5]. Of special interest is the low-density regime where chances of obtaining meaningful results are better, since the ra- tio of the scattering length to the average inter-particle distance can be used as a small parameter. Unfortunately those calculations are quickly becoming very involved since the simplest conserving approximation is the self-consistent T-matrix approach [6, 7, 8]. Alternatively, Variational-Monte-Carlo (VMC) calculations, based on the Gutzwiller wave-function [9] and QuantumMonte-Carlo (QMC) simulations have been performed [10]. These methods are providing results which then generate a need for a qualitative analytical understanding. To that aim simpler calculations based on HubbardStratonovitch decoupling of the interaction [11], slaveboson mean-field calculations (see for instance Ref. 12] and references therein), or on the moment calculation of the electronic spectral function have been performed [13]. Unfortunately the latter does not account for the damping of the quasi-particles.

The aim of this paper is to treat analytically the intermediate coupling regime, which is the most delicate. This allows us to give an analytical account of the results obtained with QMC simulations. We first review the selfconsistent T-matrix approximation. As pointed out by several authors 14, the corresponding numerical calculations typically yield a superconducting instability at a finite $T$, even in two dimensions. This contradicts the Mermin-Wagner theorem. We then propose an alternative scheme which complies with this theorem. We then proceed to the calculation of the electronic structure.

\section{(B) THEORETICAL FRAMEWORK}

We study the Hubbard model on the square lattice :

$$
H=\sum_{i, j} \sum_{\langle\sigma\rangle} t_{i j} c_{i, \sigma}^{\dagger} c_{j, \sigma}+U \sum_{i} n_{i, \uparrow} n_{i, \downarrow}
$$

We consider an attractive interaction $(U<0)$ in the intermediate coupling regime $(|U| \lesssim W), W$ being the band width. In 2 dimensions, any attractive potential 
has a bound state. In the case $|U|=W$, the binding energy $E_{b}$ has been found to be $E_{b} \approx 0.2 W$ [7], namely $E_{b} \ll W$. We are thus in a situation where bound pairs exist and have a strong influence on the physics via the splitting of the non-interacting band into 2 sub-bands. In this regime, the pairs are extended. They become purely local only in the $|U|=\infty$ limit since for any finite $U$ they can move via virtual ionization [1]. We also note that the BCS theory successfully describes the weak coupling regime. However there does not exist any analytical theory in the intermediate coupling regime, and most results are obtained out of numerical simulations [5. 10]. In the low-density regime, the self-consistent T-matrix approximation is expected to be exact and has been solved by a variety of authors [6,8],8]. Unfortunately, numerical difficulties prevented those authors from obtaining results for arbitrary $U$. We also note that the numerical solutions may lead to unphysical results such as a finite critical temperature for Bose condensation of the pairs in two dimensions, which is contradicting the MerminWagner theorem. We believe (see below) that this is due to the use of an inappropriate expression for the particle density. That however does not discredit the scheme, and we are basing our approach on it. It amounts to solving

$$
\begin{aligned}
& T\left(\vec{q}, i \nu_{n}\right)=\frac{-U}{1+U \chi\left(\vec{q}, i \nu_{n}\right)} \\
& \chi\left(\vec{q}, i \nu_{n}\right)=\beta^{-1} \sum_{\vec{p}, i \omega_{n}} G\left(\vec{p}, i \omega_{n}\right) G\left(\vec{q}-\vec{p}, i \nu_{n}-i \omega_{n}\right) \\
& \Sigma\left(\vec{q}, i \omega_{n}\right)=-\beta^{-1} \sum_{\vec{p}, i \nu_{n}} T\left(\vec{p}, i \nu_{n}\right) G\left(\vec{p}-\vec{q}, i \nu_{n}-i \omega_{n}\right) \\
& G\left(\vec{q}, i \omega_{n}\right)=\frac{1}{i \omega_{n}-t_{\vec{q}}+\mu-\Sigma\left(\vec{q}, i \omega_{n}\right)} .
\end{aligned}
$$

Here, $\omega_{n}$ are Fermionic, and $\nu_{n}$ Bosonic Matsubara frequencies. This set of equations is valid above $T_{C}$, as no anomalous Green's function enters. Otherwise one can resort to the scheme obtained by Pedersen et al., by functional derivative techniques [11]. This approximation is conserving and it diagrammatically corresponds to summing up the dressed particle-particle ladder which includes the leading order in an expansion in $k_{F} a$, 田, 8 . Another important quantity is the two-particle Green's function which is defined by :

$$
G^{(2)}\left(\vec{q}, i \nu_{n}\right)=\int_{0}^{\beta} e^{i \nu_{n} \tau}\left\langle T_{\tau}\left[Q(\vec{q}, \tau) Q^{\dagger}(-\vec{q}, 0)\right]\right\rangle d \tau,
$$

where $T_{\tau}$ is the usual time-ordering operator and the operator

$$
Q^{+}(\vec{q})=\frac{1}{N} \sum_{\vec{k}} c_{-\vec{k}, \uparrow}^{\dagger} c_{\vec{k}-\vec{q}, \downarrow}^{\dagger}
$$

creates a pair having (center-of-mass) wave vector $\vec{q}$. $G^{(2)}\left(\vec{q}, i \nu_{n}\right)$ is related to the T-matrix by :

$$
G^{(2)}\left(\vec{q}, i \nu_{n}\right)=\frac{U+T\left(\vec{q}, i \nu_{n}\right)}{U^{2}} .
$$

We calculate $G^{(2)}\left(\vec{q}, i \nu_{n}\right)$ by inserting the free-electron Green's function into expression (3) for $\chi\left(\vec{q}, i \nu_{n}\right)$. For simplicity, we approximate the density of states (DOS), $\rho(\epsilon)$, of the tight-binding band resulting from the Hamiltonian (1) by the square DOS (i.e. $\rho(\epsilon)=\frac{1}{W}$ for $|\epsilon| \leq \frac{W}{2}$ and $\rho(\epsilon)=0$ otherwise).

For small momenta, $G^{(2)}\left(\vec{q}, i \nu_{n}\right)$ is given by :

$$
\begin{aligned}
& G^{(2)}\left(\vec{q}, i \nu_{n}\right)=\frac{1}{2 W\left(1-\frac{q^{2}}{16}\right)} \times \\
& \frac{\ln \left(\frac{i \nu_{n}+\mu_{B}-\left|E_{b}\right|-2 W+\frac{q^{2} t}{2}}{i \nu_{n}+\mu_{B}-\left|E_{b}\right|-\frac{q^{2} t}{2}}\right) \ln \Phi}{\ln \Phi-\left(1+\frac{q^{2}}{16}\right) \ln \left(\frac{i \nu_{n}+\mu_{B}-\left|E_{b}\right|-2 W+\frac{q^{2} t}{2}}{i \nu_{n}+\mu_{B}-\left|E_{b}\right|-\frac{q^{2} t}{2}}\right)},
\end{aligned}
$$

where $\mu_{B}=2 \mu+W+\left|E_{b}\right|, \Phi=\frac{2 W+\left|E_{b}\right|}{\left|E_{b}\right|}$ and $\left|E_{b}\right|$ is the binding energy of a pair. The binding energy is obtained as a solution of :

$$
-\frac{1}{U}=\left.\chi\left(\vec{q}=\overrightarrow{0}, \omega=E_{b}\right)\right|_{\mu=-\frac{W}{2}},
$$

which yields

$$
\left|E_{b}\right|=2 W\left(\frac{1}{e^{\frac{-2 W}{U}}-1}\right) .
$$

The form (9) has the correct behavior for $\nu_{n}$ going to infinity in the low density regime, i.e. $G^{(2)}\left(\vec{q}, i \nu_{n}\right) \rightarrow \frac{1}{i \nu_{n}}$. The spectrum of $G^{(2)}\left(\vec{q}, i \nu_{n}\right)$ presents two features : (i) a sharp quasi-particle peak, which can be found by expanding (9) with respect to $i \nu_{n}+\mu_{B}-\frac{q^{2} t}{2}$; (ii) a continuous spectrum which extends over energies above the one of the quasi-particle. Correspondingly, the lowest order form of the T-matrix, valid for small wave vector and frequency is given by :

$$
T_{0}\left(\vec{q}, i \nu_{n}\right)=\frac{-\left|E_{b}\right|^{2} \Phi}{i \nu_{n}-\frac{\vec{q}^{2}}{4 m_{0}^{*}}+\mu_{B}} .
$$

The mass renormalization factor of a pair is given by :

$$
Z \equiv \frac{m}{m_{0}^{*}}=\frac{W+\left|E_{b}\right|}{W}-\frac{\left|E_{b}\right|^{2}}{2 W^{2}} \Phi \ln \Phi .
$$

In the intermediate coupling regime, the mass is only weakly renormalized while in the strong coupling regime, there is a strong renormalization of the order $\frac{W}{|U|}$. Due to the relationship between the two-particle T-matrix and the two-particle Green's function, the quantity $\mu_{B}$ that we defined above does represent the chemical potential 
of a pair, which has bosonic character.

For $\vec{q}$ 's close to the nesting vector $\vec{Q}=(\pi, \pi)$, we obtain:

$$
T_{0}\left(\vec{q}, i \nu_{n}\right)=\frac{-U^{2}}{i \nu_{n}+\frac{(\vec{q}-\vec{Q})^{2}}{4 m^{*}}+2 \mu+|U|} .
$$

In the vicinity of the zone corner, the renormalization of the pair-mass is different from the one close to the zone center. Even in the intermediate coupling regime, it is strongly renormalized to be $\frac{m^{*}}{m} \approx \frac{|U|}{t}$. At $\vec{q}=\vec{Q}$, the form (9) of the T-matrix is actually exact, related to the fact that the creation operator

$$
\eta^{\dagger}=\sum_{\vec{p}} c_{\vec{p}+\vec{Q}, \uparrow}^{\dagger} c_{-\vec{p}, \downarrow}^{\dagger}
$$

of an " $\eta$-pair" with center of mass momentum $\vec{Q}$, satisfies the simple commutation relation [15,16,17]:

$$
\left[H, \eta^{\dagger}\right]=(U-2 \mu) \eta^{\dagger} .
$$

Using the above found expressions (12) and (14), we can calculate the self-energy. To lowest order, we insert the free-electron Green's function in Eqn. (4). The first contribution to the self-energy arises from the poles of the T-matrix. Due to the statistical factors we obtain (to that order of approximation) that the contribution of the $\eta$-resonance is exponentially small, as well as those following from the poles of the Green's function. After performing analytical continuation, we are left with :

$$
\Sigma_{1}(\vec{k}, \omega)=\frac{U^{2} n_{d}}{\omega+t_{\vec{k}}-\mu+\mu_{B}+i 0^{+}} .
$$

The quantity $n_{d}$ will be defined below, in Eqns. (26) and (27). $\Sigma_{1}(\vec{k}, \omega)$ yields then the Green's function as :

$$
\begin{aligned}
& G(\vec{k}, \omega)= \\
& \quad \frac{1}{2}\left(1+\frac{2\left(t_{\vec{k}}-\mu\right)-\mu_{B}}{x_{\vec{k}}}\right) \frac{1}{\omega+\frac{1}{2} \mu_{B}-\frac{1}{2} x_{\vec{k}}+i 0^{+}} \\
& +\frac{1}{2}\left(1-\frac{2\left(t_{\vec{k}}-\mu\right)+\mu_{B}}{x_{\vec{k}}}\right) \frac{1}{\omega+\frac{1}{2} \mu_{B}+\frac{1}{2} x_{\vec{k}}+i 0^{+}},
\end{aligned}
$$

where $x_{\vec{k}}=\sqrt{\left(2\left(t_{\vec{k}}-\mu\right)+\mu_{B}\right)^{2}+4 U^{2} n_{d}}$. We immediately note the two limiting behaviors, with respect to momentum $\vec{k}$ :

$$
x_{\vec{k}} \approx \Delta+2 t \gamma k^{2},
$$

with $\gamma=\frac{\left|E_{b}\right|}{\Delta}$ and

$$
\Delta=\sqrt{\left|E_{b}\right|^{2}+4 U^{2} n_{d}}
$$

for small momenta; respectively,

$$
x_{\vec{k}} \approx 2\left(t_{\vec{k}}-\mu\right)+\mu_{B}+\frac{2 U^{2} n_{d}}{2\left(t_{\vec{k}}-\mu\right)+\mu_{B}},
$$

for large momenta.

At this stage of the calculation, the Green's function has a two-pole structure. The lower excitation branch corresponds to quasi-bound fermions (hereafter denoted as "bosonic" band), while the upper branch describes the unpaired fermions (fermionic band). At small momenta, we obtain:

$$
\begin{aligned}
G(\vec{k}, \omega) & =\frac{\Delta+\left|E_{b}\right|}{2 \Delta} \frac{1}{\omega+\frac{1}{2}\left(\mu_{B}-\Delta\right)-\gamma t k^{2}+i 0^{+}} \\
& +\frac{\Delta-\left|E_{b}\right|}{2 \Delta} \frac{1}{\omega+\frac{1}{2}\left(\mu_{B}+\Delta\right)+\gamma t k^{2}+i 0^{+}}
\end{aligned}
$$

with the spectral weight mainly located in the unpaired fermion band (first contribution in Eqn. (22)). At large momenta, the Green's function results into :

$$
\begin{aligned}
G(\vec{k}, \omega) & =\frac{1-\frac{2 U^{2} n_{d}}{\left(2\left(t_{\vec{k}}-\mu\right)+\mu_{B}\right)^{2}}}{\omega-\left(t_{\vec{k}}-\mu\right)+i 0^{+}} \\
& +\frac{\frac{2 U^{2} n_{d}}{\left(2\left(t_{\vec{k}}-\mu\right)+\mu_{B}\right)^{2}}}{\omega+t_{\vec{k}}-\mu+\mu_{B}+i 0^{+}},
\end{aligned}
$$

where the weight of the paired fermion band is even smaller than for small momenta. The form of the Green's function Eqn. (18) differs from the one of Ref. 19] because the chemical potential $\mu$ is located below the fermionic band in our problem.

We note that there are two equivalent expressions for the particle density operator $\hat{n}$ :

$$
\hat{n}_{i}=\sum_{\sigma}\left(\hat{n}_{i, \sigma}\left(1-\hat{n}_{i,-\sigma}\right)+\hat{n}_{i, \sigma} \hat{n}_{i,-\sigma}\right)
$$

On one hand, we can use the left hand side to express the particle density $n$ as $n_{1}$, where the subscript 1 indicates that the density is calculated out of the one-particle Green's function :

$$
n_{1}=\beta^{-1} \sum_{i \omega_{n}, \vec{k}} \sum_{\sigma} G_{\sigma}\left(\vec{k}, i \omega_{n}\right) e^{i \omega_{n} 0^{+}} .
$$

Alternatively, we may use the r.h.s. of Eqn. (24) by separating explicitly the contributions from the unpaired fermions (first term) and the doubly occupied sites (second term). The total density $n_{d}$ of the latter is given by

$$
\begin{aligned}
n_{d} & =\frac{1}{\beta} \sum_{\vec{q}} \sum_{i \nu_{n}} G^{(2)}\left(\vec{q}, i \nu_{n}\right) e^{i \nu_{n} 0^{+}} \\
& =\sum_{\vec{q}} \int_{-\infty}^{\infty} \frac{d \omega}{2 \pi} \operatorname{Im}\left\{G^{(2)}\left(\vec{q}, \omega+i 0^{+}\right)\right\} N_{B}(\omega),
\end{aligned}
$$


$N_{B}(\omega)$ being the Bose-Einstein distribution function. Owing to the latter, at low temperatures, only the low-energy part of the two-particle spectrum, $\operatorname{Im}\left\{G^{(2)}\left(\vec{q}, \omega+i 0^{+}\right)\right\}$, will contribute to $n_{d}$. According to the discussion following Eqn. (11), this low-energy part has two contributions : the sharp quasi-particle excitation, given by (12), which dominates for small momenta, and the low-frequency tail of the continuous spectrum of $G^{(2)}\left(\vec{q}, i \nu_{n}\right)$. Since it does not have a sharp structure, it would only contribute a featureless background to the one-electron spectral function; thus, we have neglected it for the calculation of the self-energy $\Sigma_{1}(\vec{k}, \omega)$. Neglecting the continuum also in calculating $n_{d}$, we find, according to Eqns. (8), (12) and (26) :

$$
n_{d}=R \sum_{\vec{q}} N_{B}\left(\frac{q^{2}}{4 m} Z-\mu_{B}\right) .
$$

Thus, in our approximation, the number of doubly occupied sites $n_{d}$ is given by the "number of bosons", $n_{B}$

$$
n_{B} \equiv \sum_{\vec{q}} N_{B}\left(\frac{q^{2}}{4 m} Z-\mu_{B}\right)
$$

weighted by a factor

$$
R=\left(\frac{\left|E_{b}\right|}{2 W}\right)^{2} \Phi(\ln \Phi)^{2}
$$

which is the residue of the two-particle Green's function at the bottom of the two-particle band. These bosons, having energy $E_{B}=\frac{q^{2}}{4 m} Z$ and chemical potential $\mu_{B}$, represent pairs of electrons being (virtually) bound by the on-site attraction. These results correspond to the observation of other authors (see, for example Ref. [8]) that, for sufficiently strong attraction, the two-particle Green's function, (respectively the T-matrix) can be interpreted as a "bosonic Green's function". However, there is a weight factor between the two. For large $|U|$, this weight factor $R$ goes to one : in the strong coupling limit, all the double occupancy is due to coherently propagating quasi-bound pairs. In the intermediate coupling regime, which we want to consider $(|U| \lesssim W)$, the weight factor $R$ is roughly 0.5 : only about one half of the two-particle spectrum in Eqn. (9) is resulting from coherent excitations. In the weak coupling regime, $R$ vanishes, thus rendering our approximation invalid in this limit.

Since, in our approach, there is gap in the one-electron spectrum, separating the "bosonic" band from the fermionic one, there is no difficulty to obtain the number of unpaired electrons $n_{F}$ as :

$$
n_{F}=\sum_{\vec{k}, \sigma} \Xi_{\vec{k}} f_{F}\left(\varepsilon_{\vec{k}, \sigma}\right),
$$

where $f_{F}\left(\varepsilon_{\vec{k}, \sigma}\right)$ is the usual Fermi distribution function. The dispersion $\varepsilon_{\vec{k}, \sigma}$ of the unpaired fermions and the spectral weight $\Xi_{\vec{k}}$ entering Eqn. (30) are :

$$
\begin{aligned}
\varepsilon_{\vec{k}, \sigma} & =\frac{1}{2}\left(x_{\vec{k}}-\mu_{B}\right), \\
\Xi_{\vec{k}} & =\frac{1}{2}\left(1+\frac{2\left(t_{\vec{k}}-\mu\right)-\mu_{B}}{x_{\vec{k}}}\right) .
\end{aligned}
$$

The total particle density results into :

$$
n=n_{F}+2 n_{d} .
$$

For non-interacting particles, we may equally well use both r.h.s. and l.h.s of Eqn. (24) to calculate the density, since Wick's theorem applies. However, the identity (24) may be violated in an approximate treatment like perturbation theory. This is the reason why the self-consistent T-matrix calculation breaks down at low $T$ in two dimensions. By calculating the density by means of Eqn. (25), there is nothing preventing $T(\vec{q}=\overrightarrow{0}, \omega=0)$ from diverging at finite $T$, signaling a phase transition, while using Eqn. (32) would definitively keep $T(\overrightarrow{0}, 0)$ finite for any finite temperature. By making use of Eqn. (32), we make sure that Bose condensation can only take place at $T=0$, in agreement with the Mermin-Wagner theorem. Indeed, according to the expressions (27) and (28) for $n_{d}$ and $n_{B}$, respectively, the bosonic chemical potential $\mu_{B}$, for a given $n_{d}$ (or $n_{B}$ ) and for $d=2$, is different from zero at any finite temperature, which inhibits Bose condensation, except for $T=0$. In principle, for $d=2$ one should see a Kosterlitz-Thouless (KT) transition. However, since our approximation does not treat the bosonic phase fluctuations in an adequate way, we cannot expect to see the KT-scenario. On the other hand, $T_{C}$ may well be finite for a 3-d system. Actually our procedure is similar in spirit to the two-particle self-consistent approach to the repulsive Hubbard model by Vilk et al. 118.19].

Now for a two-dimensional system, we can explicitly evaluate the number of pairs. We obtain :

$$
\begin{aligned}
& n_{d}=\frac{2 R}{\beta W Z} \ln \left(\frac{e^{-\beta\left(W \frac{Z}{2}-\mu_{B}\right)}-1}{e^{\beta \mu_{B}}-1}\right), \\
& n_{F}=\frac{1}{\beta W \gamma}\left(\frac{\left|E_{b}\right|+\Delta}{\Delta}\right) \ln \left(\frac{e^{\frac{\beta}{2}\left(\mu_{B}-\Delta\right)}+1}{e^{\frac{\beta}{2}\left(\mu_{B}-\Delta-\gamma W\right)}+1}\right),
\end{aligned}
$$

where $\Delta$ and $\gamma$ have been given above.

We are now ready to (numerically) solve Eqns. (32), (33) and (34) for the chemical potential, as a function of temperature. Hereafter, the density $n$ is fixed to be $n=0.1$. The resulting $\mu_{B}(T)$ vanishes exponentially at $T=0$. From Eqns. (8) and (12), we obtain that the range of the two-particle Green's function, $\xi$, is given by

$$
\frac{\xi}{a}=\sqrt{\frac{t}{2\left|\mu_{B}\right|}},
$$

where $a$ is the lattice spacing. It is displayed on fig. 1, for three different values of the coupling strength $U$. We 
note that it shows a strong dependence on $U$. At very low temperatures, it is given by :

$$
\frac{\xi}{a}=\sqrt{\frac{\beta t}{2}} e^{\frac{\beta W Z_{n}}{8 R}} .
$$

Oppositely, in the intermediate temperature range, $\xi$ becomes independent of $U$. We also find that $\xi$ decreases with increasing $|U|$. In the strong coupling limit the ratio $\frac{Z}{R}$ tends to zero and thus the exponential divergence of $\xi$ in $\frac{1}{T}$ is suppressed (there remains only the power-law dependence $\left.\xi \sim \frac{1}{\sqrt{T}}\right)$.

We may now define a coherence temperature $T_{c o h}$ as the temperature at which the range of the two-particle Green's function exceeds 10 lattice spacings. We obtain $T_{c o h} \approx 0.16 t$ for $U=-4 t$ and $T_{c o h} \approx 0.1 t$ for $U=-6 t$, which may be compared to $T_{C}$ as obtained from the numerical simulations $[10]$. We see that they compare favorably and that, moreover, $T_{c o h}$ decreases with increasing $U$. Finally, we note that $\xi$ becomes of the order of the lattice spacing at temperatures well below $\left|E_{b}\right| / 2$. We now turn to the temperature dependence of the number of pairs. It is displayed on fig. 2, as a function of $T$, for three different values of $U$. At low temperatures, it is independent of $T$. Since the binding energy of the pair (and the gap) decreases as $U$ gets smaller, $n_{d}$ begins to decrease at a lower $T$ for $U=-6 t$ than for $U=-10 t$, for example. In all cases $n_{d}$ decreases by a factor 2 at $T \approx \Delta / 2$.

In order to reach a better self-consistency, we now calculate how the quasi-bound states affect the two-particle propagator. Introducing $G_{1}(\vec{q}, \omega)$ as the lower branch of $G(\vec{q}, \omega)$ given by Eqn. (18), we can calculate the first correction to the two-particle propagator as $\chi_{1}$, with :

$$
\begin{aligned}
\chi_{1}\left(\vec{q}, i \nu_{n}\right) & =\sum_{i \omega_{n}, \vec{p}} G_{1}\left(\vec{q}-\vec{p}, i \nu_{n}-i \omega_{n}\right) G_{0}\left(\vec{p}, i \omega_{n}\right) \\
& +\sum_{i \omega_{n}, \vec{p}} G_{0}\left(\vec{q}-\vec{p}, i \nu_{n}-i \omega_{n}\right) G_{1}\left(\vec{p}, i \omega_{n}\right) .
\end{aligned}
$$

Carrying out the summation over Matsubara frequencies, we obtain that the statistical factors are exponentially small and thus there is no correction to $\chi$ to that order. Thus, the T-matrix is still given by Eqn. (12). We can now proceed to the calculation of the second-order correction to the self-energy. It is given by :

$$
\Sigma_{2}\left(\vec{q}, i \omega_{n}\right)=\sum_{i \nu_{n}, \vec{p}} T_{0}\left(\vec{p}, i \nu_{n}\right) G_{1}\left(\vec{p}+\vec{q}, i \nu_{n}+i \omega_{n}\right),
$$

yielding, after performing analytical continuation,

$$
\begin{aligned}
& \Sigma_{2}(\vec{q}, \omega)= \\
& \left(\frac{\left|E_{b}\right|^{2} \Phi t}{W \gamma}\right)\left(\frac{x_{\vec{q}}-2\left(t_{\vec{q}}-\mu\right)-\mu_{B}}{2 x_{\vec{q}}}\right)
\end{aligned}
$$

$$
\begin{aligned}
& \times\left(\frac{1}{\omega-\frac{1}{2}\left(x_{\vec{q}}-\mu_{B}\right)+i 0^{+}}\right) \\
& +\frac{U^{2} \omega_{c}}{W}\left(\frac{x_{\vec{Q}-\vec{q}}-2\left(t_{\vec{Q}-\vec{q}}-\mu\right)-\mu_{B}}{2 x_{\vec{Q}-\vec{q}}}\right) \\
& \times\left(\frac{1}{\omega+2 \mu+|U|-\frac{1}{2}\left(x_{\vec{Q}-\vec{q}}+\mu_{B}\right)+i 0^{+}}\right),
\end{aligned}
$$

where $\omega_{c}$ is a frequency cut-off needed by the assumption that the $\eta$-resonance is sharp for $\frac{(\vec{Q}-\vec{q})^{2}}{4 m^{*}} \leq \omega_{c} \approx \frac{U^{2}}{W}$, with $m^{*}=m \frac{|U|}{t}$. This corresponds to $|\vec{Q}-\vec{q}| \lesssim \frac{U}{W}$, as it is borne out by numerical calculations of the two-particle Green's function $G^{(2)}$ covering the whole Brillouin zone [11, 17]. The precise value of the cut-off has little influence in the following (numerical) results. We note that the first contribution in Eqn. (39) is following from the longwavelength behavior of the T-matrix, while the second is due to the $\eta$-resonance. Even though both $\Sigma_{1}$ and the first contribution to $\Sigma_{2}$ originate from the pole of $T_{0}\left(\vec{q}, i \nu_{n}\right)$, given in Eqn. (12), they are found to have opposite dispersions. The total self-energy results as :

$$
\Sigma(\vec{q}, \omega)=\Sigma_{1}(\vec{q}, \omega)+\Sigma_{2}(\vec{q}, \omega) .
$$

At this point of the calculation, we can recalculate the particle density $n_{1}$ by evaluating explicitly Eqn. (25), using the Green's function resulting from Eqn. (40). Since there is no a priori reason that this would yield a result comparable to what is following from Eqn. (32), this is a consistency check of the framework we are using. The result is displayed on fig. 3 . It is obvious that this is in very good agreement with the expected value $n=0.1$ for all values of $U$. This means that our calculation is consistent. It also implies that, on one hand a small change in the chemical potential corresponds to a small change in the particle density, but on the other hand, it may induce a big change in the pair density.

\section{(C) EXCITATION SPECTRUM}

We can now summarize our findings by plotting the various pieces of the spectral function. This is done on fig. 4a, for $U=-6 t\left(E_{b}=-t\right)$ and on fig. $4 \mathrm{~b}$ for $U=-10 t\left(E_{b}=-4.2 t\right)$ for momenta along the diagonal of the Brillouin zone. In both cases, the spectrum consists of four branches. The spectral weights of the various branches are displayed on fig. 5a for $U=-6 t$ and fig. $5 \mathrm{~b}$ for $U=-10 t$.

These results can be commented as follows :

(i) There is a branch at negative energies following from 
the quasi-bound states. It is centered around $-\frac{W}{2}-$ $\frac{\Delta+\left|E_{b}\right|}{4}$ with a width $W-\frac{\Delta-\left|E_{b}\right|+2 \mu_{B}}{2}<W$. The dependence in $U$ is weak, and the dispersions for $|U|=6 t$ and $|U|=10 t$ are essentially the same, up to a small shift; moreover, we note that they are opposite to the free fermion dispersion. The weight decreases with increasing momentum and gets mostly negligible for $q_{x} \sim 2$. It decreases with increasing $|U|$, at small $\vec{q}$ 's, but the total weight remains constant.

(ii) The fermionic band that was coming out of the first approximation (Eqn. (18)) is now resulting as a superposition of two branches which would merge into a single one, were damping taken into account. This superposition is done out of the two branches which are most free-electron-like. This is somewhat arbitrary, especially in the domain where the hybridization with the other branch, which lies at positive energies too (see below), is strong. Consequently, we do not show the spectral weights in this range. The width of the fermionic band is slightly larger than the original bandwidth, and shows little dependence on $U$. It lies at slightly larger energies for $|U|=10 t$ than for $|U|=6 t$, but again the dependence in $U$ is weak. Its dispersion is parallel to the free fermion one.

(iii) There is a third branch resulting from the $\eta$ - resonance; its dependence in $U$ is weak and both curves (for $|U|=6 t$ and $|U|=10 t$ ) are parallel. It comes down in energy with increasing $|U|$. The contribution of the $\eta$-resonance band is very small, barely accounting for 5 percent of the spectral weight at its maximum, which is located at $\vec{q}=\vec{Q}$.

It is interesting to compare our results with Quantum Monte Carlo (QMC) simulations 10,20. The calculations of Ref. [10] have been performed for $n=0.4$ and for $|U|=-4 t,-8 t,-12 t$. This is likely to be outside the realm of densities for which the T-matrix approximation is really valid. Nevertheless, the following features coincide with our findings : there are two distinct excitation branches which get more and more clearly separated by a gap when $|U|$ increases. The width of the two bands is smaller than in our calculation, in particular the lower (bosonic) band has very little dispersion. However, the weights (the fermionic band has large weight for large wave vectors, whereas the weight of the negative energy bosonic band is concentrated near the zone center) is similar to our figures 4 and 5 .

Ref. [20] presents new results for the one-electron spectral function for $n=0.1$, the same value that we have used, and $|U|=8 t$. Here again, there is a fermionic branch at positive energies $E$ (positive with respect to the chemical potential), separated by a "pseudo-gap" from the excitations at $E<0$. Weight and width of the fermionic band is similar to our result. The excitations at $E<0$ have most weight near the zone center, as we find it. However, their structure seems to be more complex : with increas- ing wave number they split into two "sub-branches". The lower part has downward dispersion and seems to correspond to our bosonic branch. The other sub-branch produces weight near the chemical $(E \simeq 0)$ potential for large wave numbers. This might correspond to our $\eta$ resonance, provided that its energy near the zone corner, lying at a positive energy in our calculation, would in reality be lower. In this respect, the spectral functions for $n=0.4$ and $|U|=-6 t$, also shown in Ref. 20], are particularly interesting : for $E>0$, besides the strong fermionic branch, there is a second branch of excitations the dispersions of which is very similar to the $\eta$-peak in our figures 4 and 5 .

Thus, the main features of our spectral functions seem to be present in the Monte Carlo results, although the latter show a more complex structure. A detailed and more quantitative comparison with QMC should also take into account the fact the "Maximum Entropy method" used there in order to extract spectral functions from data obtained as functions of the (imaginary) Matsubara frequencies does not easily allow for an unambiguous identification of excitations with small weight.

Finally, we note that we do not obtain any spectral weight at zero frequency, signaling the presence of a correlation induced gap. We also checked that including particle-hole excitations in the calculation does not affect this conclusion. The appearance of a true gap in our calculation is, at least partly, due to the fact that our spectral lines have no width (except that the "doubling" of the fermionic branch gives a hint to a broadening of the latter). Spectral functions with finite line width would be obtained either by doing the $\vec{q}$-sums in the expression (14) for the self-energy more precisely, and/or by evaluating $G$ and $\Sigma$ by solving Eqns. (2) to (5) self-consistently.

In summary, we have determined the excitation spectrum of the attractive Hubbard model at intermediate coupling out of a simple analytical calculation. We first pointed out an intrinsic problem of perturbation theory relative to the implementation of the Mermin-Wagner theorem. We made use of an alternative expression for the density to obtain a qualitatively correct theory which does not break down at low $T$ in two dimensions, in contrast to previous self-consistent calculations. We obtain an analytical expression for the Green's function which reproduces the qualitative features of the QMC simulations in the low density regime.

We acknowledge valuable discussions with T. Schneider, J.M. Singer, M.H. Pedersen and J.J. RodríguezNúñez. We thank J.M. Singer for providing us with his numerical data prior to publication. This work was financially supported by the Swiss National Science Foundation, under grants 20-43111.95 and 20-47149.96. M. Yu. K. acknowledges the University of Neuchâtel, where part of this work has been performed, for hospitality and the Swiss National Science Foundation for partial funding. 
[1] P. Nozières and S. Schmitt-Rink, J. Low Temp. Phys. 59, 195 (1985).

[2] M. Drechsler and W. Zwerger, Ann. Phys. 1, 15 (1992).

[3] Th. Meintrup, T. Schneider and H. Beck, Europhys. Lett. 31, 231 (1995).

[4] V.M. Galitskii, Sov. Phys. JETP 7, 104 (1958); D.M. Eagles, Phys. Rev. 186, 456 (1969); P. Bloom, Phys. Rev. B 12, 125 (1975)

[5] J.R. Engelbrecht and M. Randeria, Phys. Rev. Lett 65, 1032 (1990); H. Fukuyama, Y. Hasegawa and O. Narikiyo, J. Low Temp. Phys. 60, 2013 (1991).

[6] R. Frésard, B. Glaser and P. Wölfle, J. Phys.: Cond. Mat. 4, 8565 (1992).

[7] R. Micnas, M.H. Pedersen, S. Schafroth, T. Schneider, J.J. Rodríguez-Núñez and H. Beck, Phys. Rev. B 52, 16223 (1995).

[8] R. Haussmann, Z. Phys. B 91, 291 (1993).

[9] P.J.H. Denteneer, G. An and J.M.J. van Leeuwen, Phys. Rev. B 47, 6256 (1993).

[10] J.M. Singer, M.H. Pedersen, T. Schneider, H. Beck and H.G. Matuttis, Phys. Rev. B 54, 1286 (1996); J.M. Singer, M.H. Pedersen and T. Schneider, submitted to Physica C.

[11] M.H. Pedersen, Ph.D. Thesis, University of Zurich (1996); M.H. Pedersen, J.J. Rodríguez-Núñez, H. Beck, T. Schneider and S. Schafroth, accepted for publication in Z. Phys. $B$ and cond-mat/9702173.

[12] B. Bulka, cond-mat/9703040.

[13] T. Schneider, M.H. Pedersen and J.J. Rodríguez-Núñez, Z. Phys. B 100, 263 (1996).

[14] See for example, S. Schafroth, J. J. Rodríguez-Núñez and H. Beck, J. Phys.: Cond. Mat. 9, L111 (1997).; J.J. Rodríguez-Núñez, private communication.

[15] C.N. Yang, Phys. Rev. Lett. 63, 2144 (1989)

[16] E. Nowak, Z. Phys. B 45, 173 (1981)

[17] E. Demler, S. C. Zhang, N. Bulut and D.J. Scalapino, Int. J. of Mod. Phys. B 10, 2137 (1996).

[18] Y.M. Vilk, L. Chen and A.-M.S. Tremblay, Phys. Rev. B 49, 13267 (1994) and Physica C 235-240, 2235 (1994).

[19] Y.M. Vilk and A.-M.S. Tremblay, Europhys. Lett 33, 159 (1996) and cond-mat/9702188.

[20] J.M. Singer and T. Schneider, private communication.

FIG. 1. Range of the two-particle Green's function, as a function of temperature $T$, at density $n=0.1$, for three different interaction strengths, $U=-4 t$ (short-dashed line), $U=-6 t$ (full line), $U=-8 t$ (dotted line) and $U=-10 t$ (long-dashed line).

FIG. 2. Density of quasi-bound pairs as a function of temperature $T$, at density $n=0.1$, for $U=-6 t$ (full line), $U=-8 t$ (dotted line) and $U=-10 t$ (dashed line).

FIG. 3. Particle density $n_{1}$ which is obtained by using Eqn. (25); the chemical potential is calculated numerically out of Eqn. (32).
FIG. 4. a) Spectrum of the Green's function at $n=0.1$, $T / t=0.1$ and $U=-6 t$ It consists of the bosonic band (dashed line), the fermionic bands (dotted and dashed-dotted lines) and the $\eta$-resonance band (full line). b) The same for $U=-10 t$.

FIG. 5. a) Spectral weight of the bosonic band (dashed line), the fermionic bands (dotted line) and the $\eta$-resonance band (full line), for $n=0.1, T / t=0.1$ and $U=-6 t$. b) The same for $U=-10 t$. 


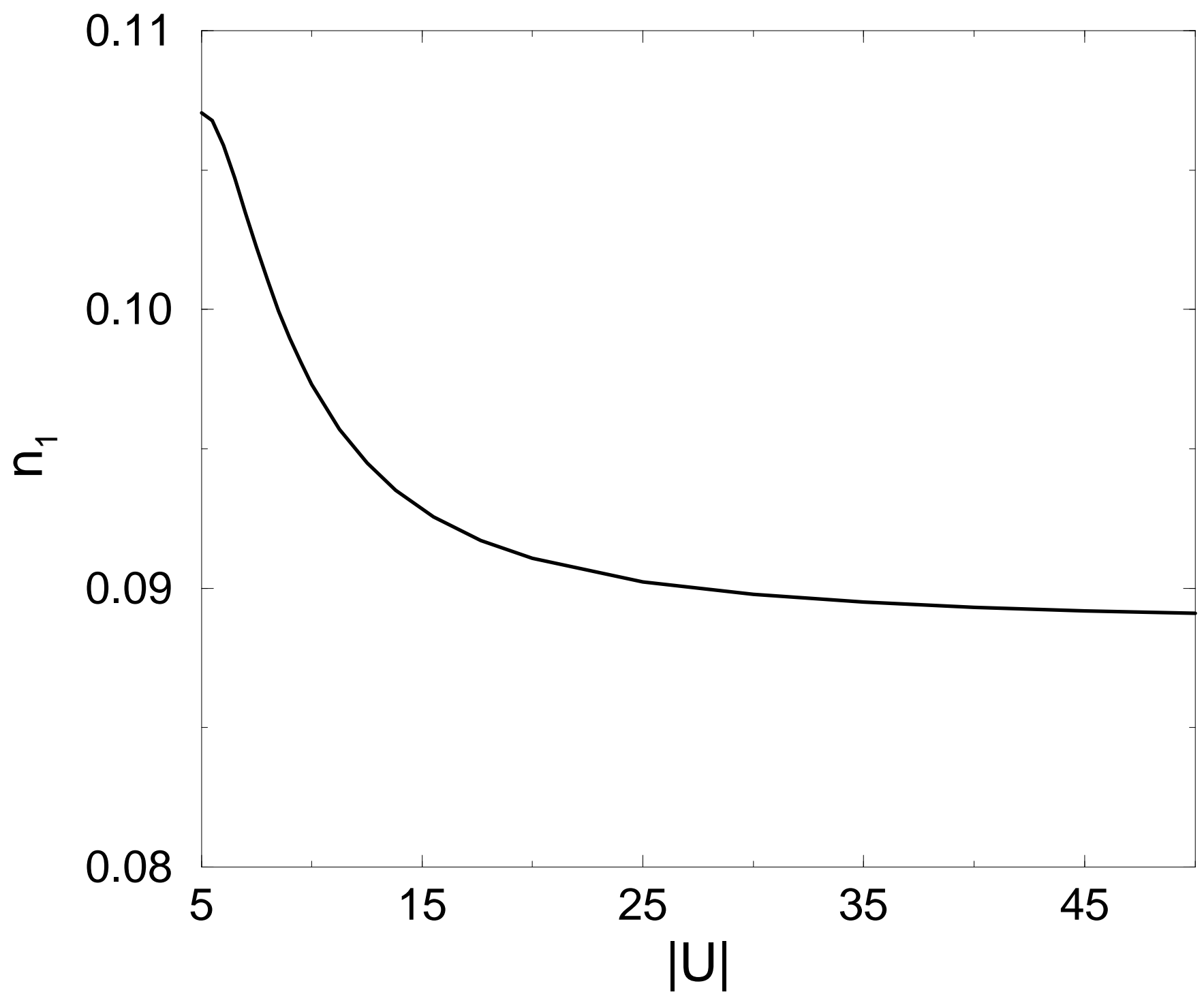


Dispersion Relations

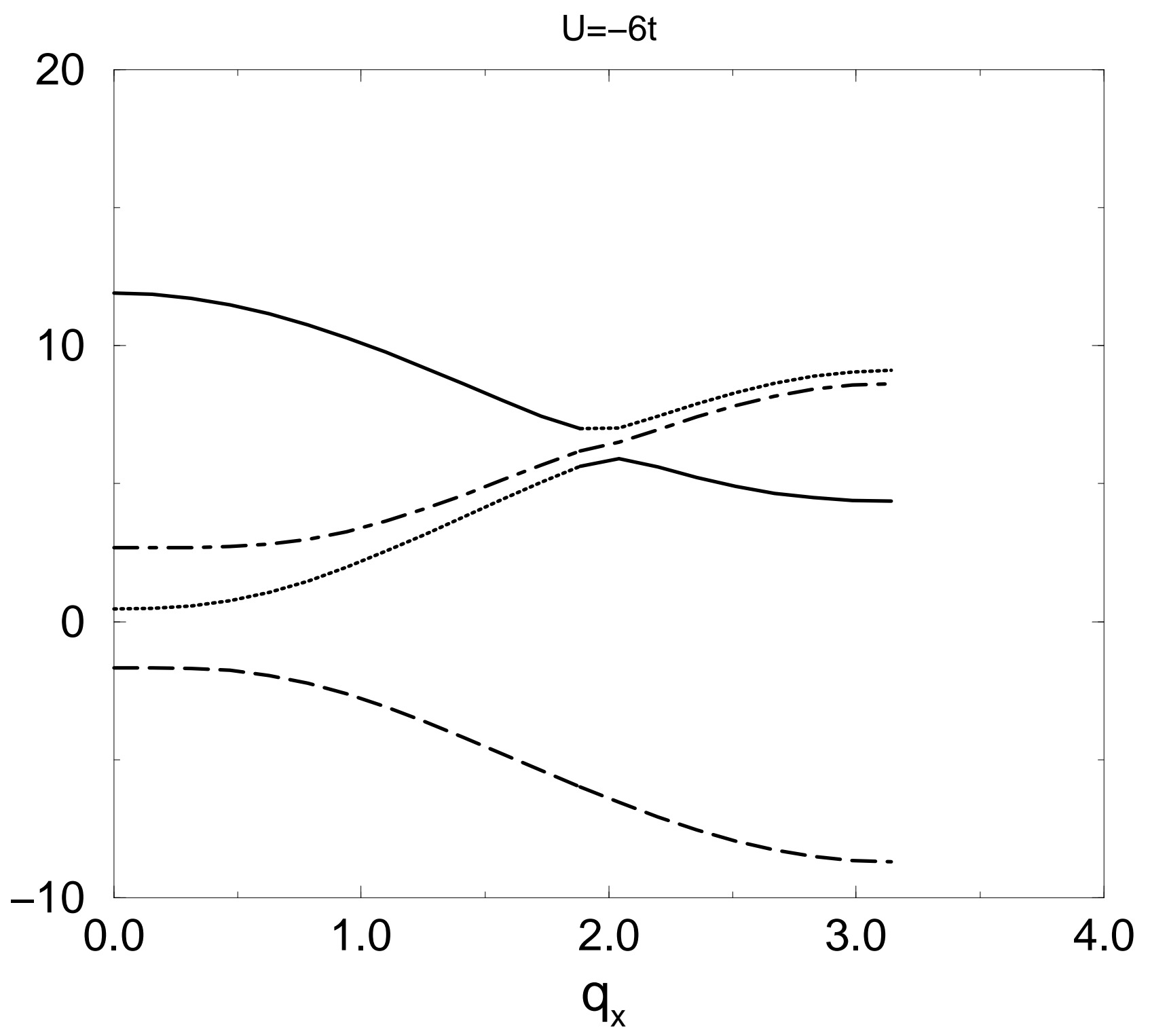




\section{Spectral Weights}

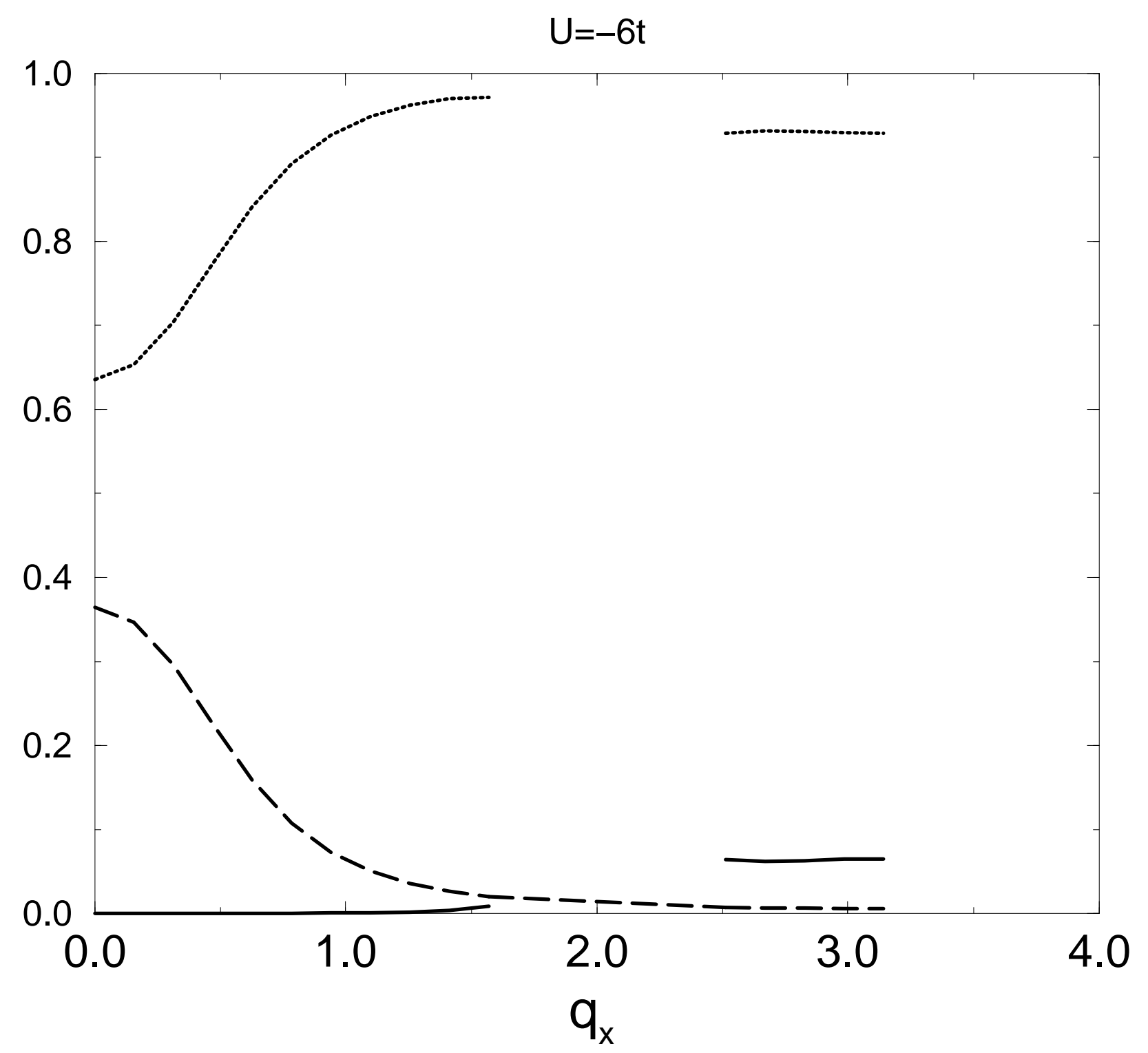


Dispersion Relations

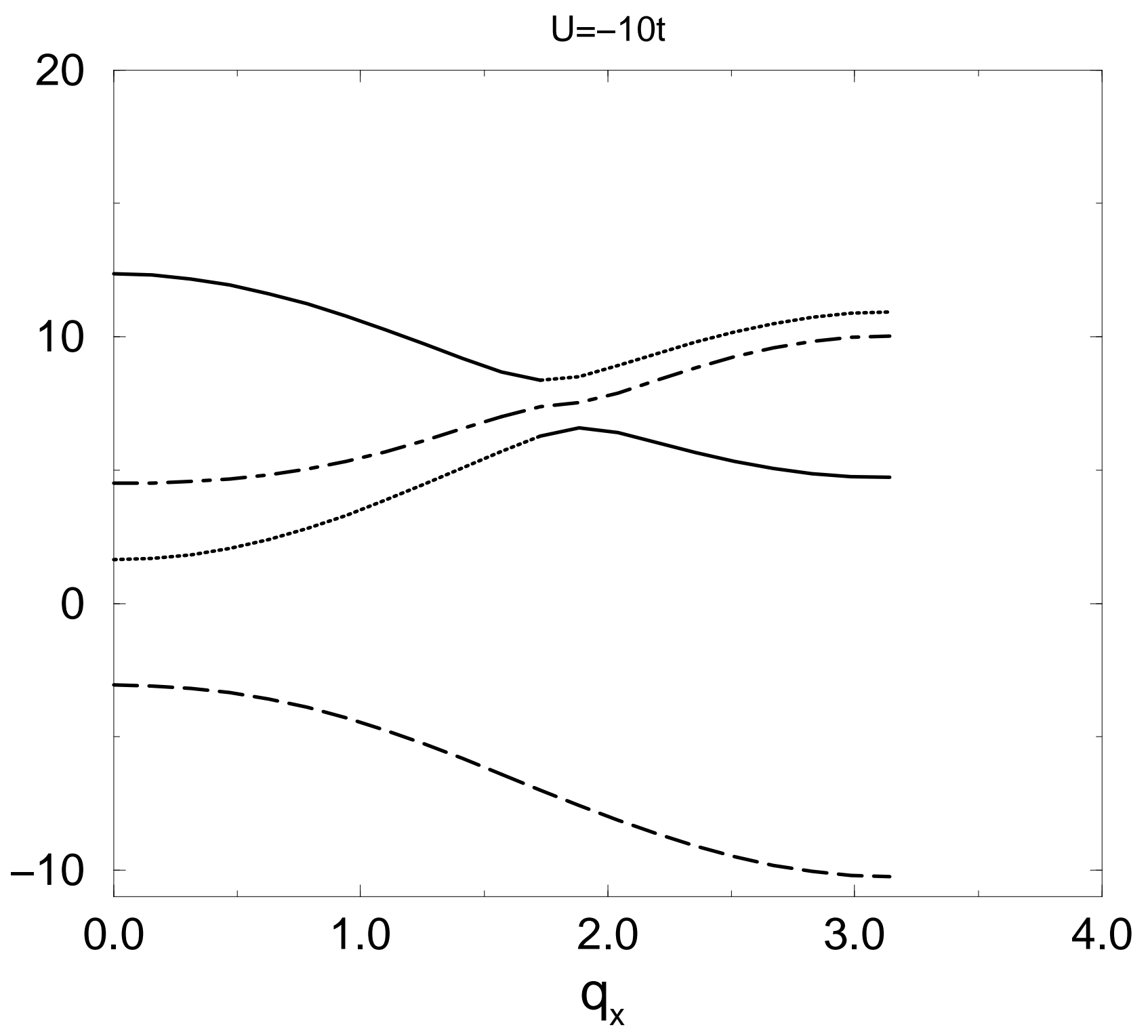




\section{Spectral Weights}

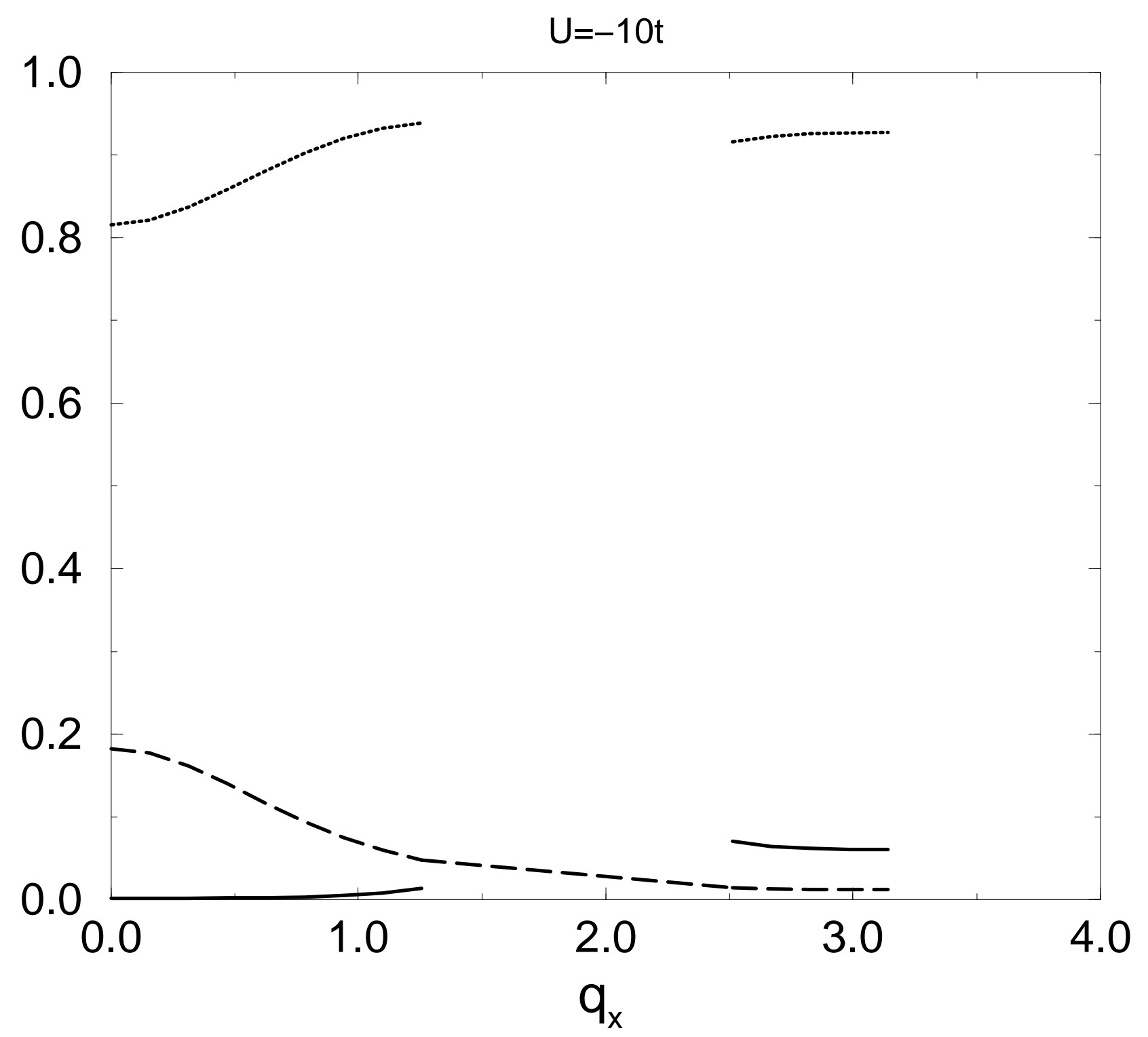




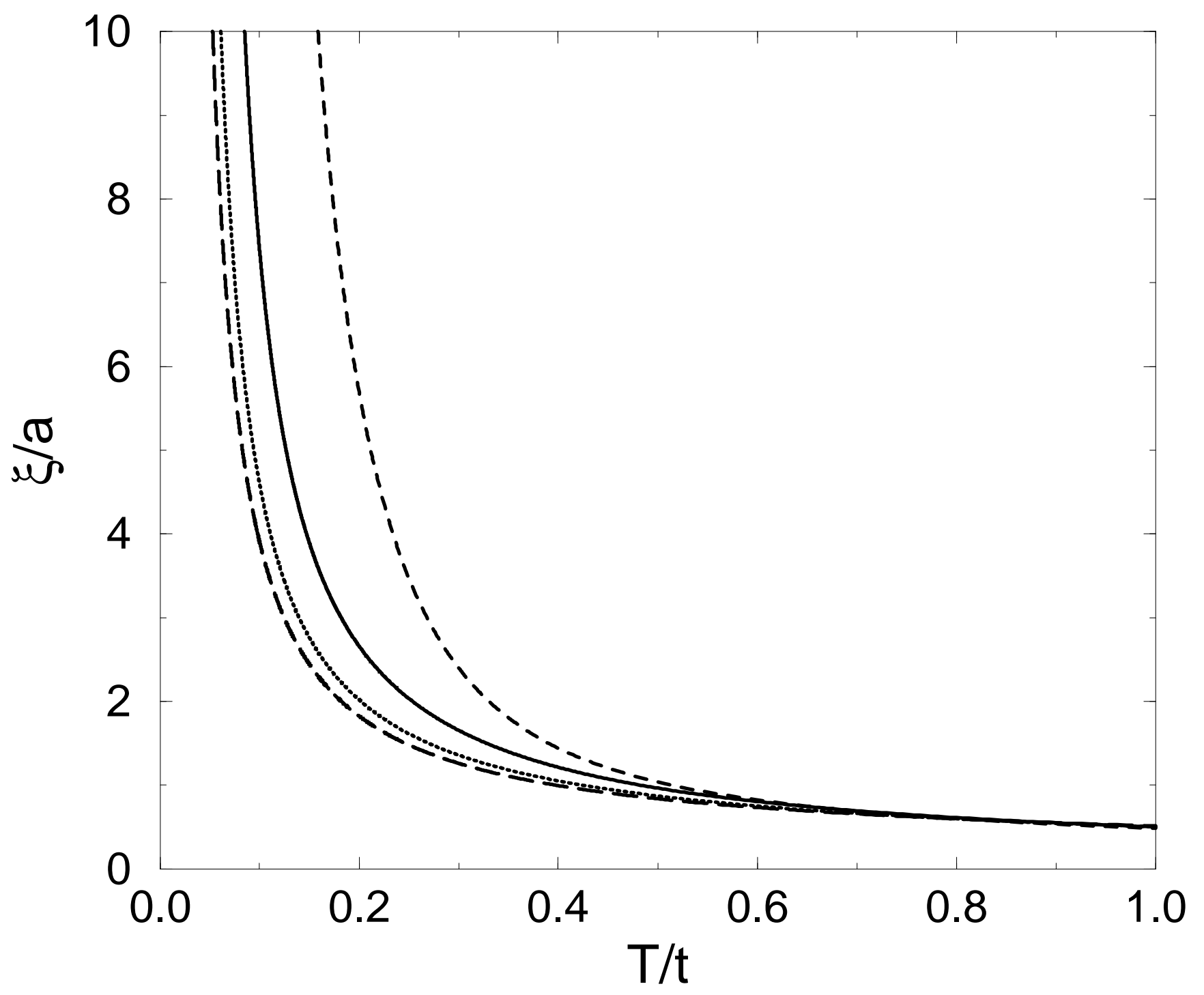




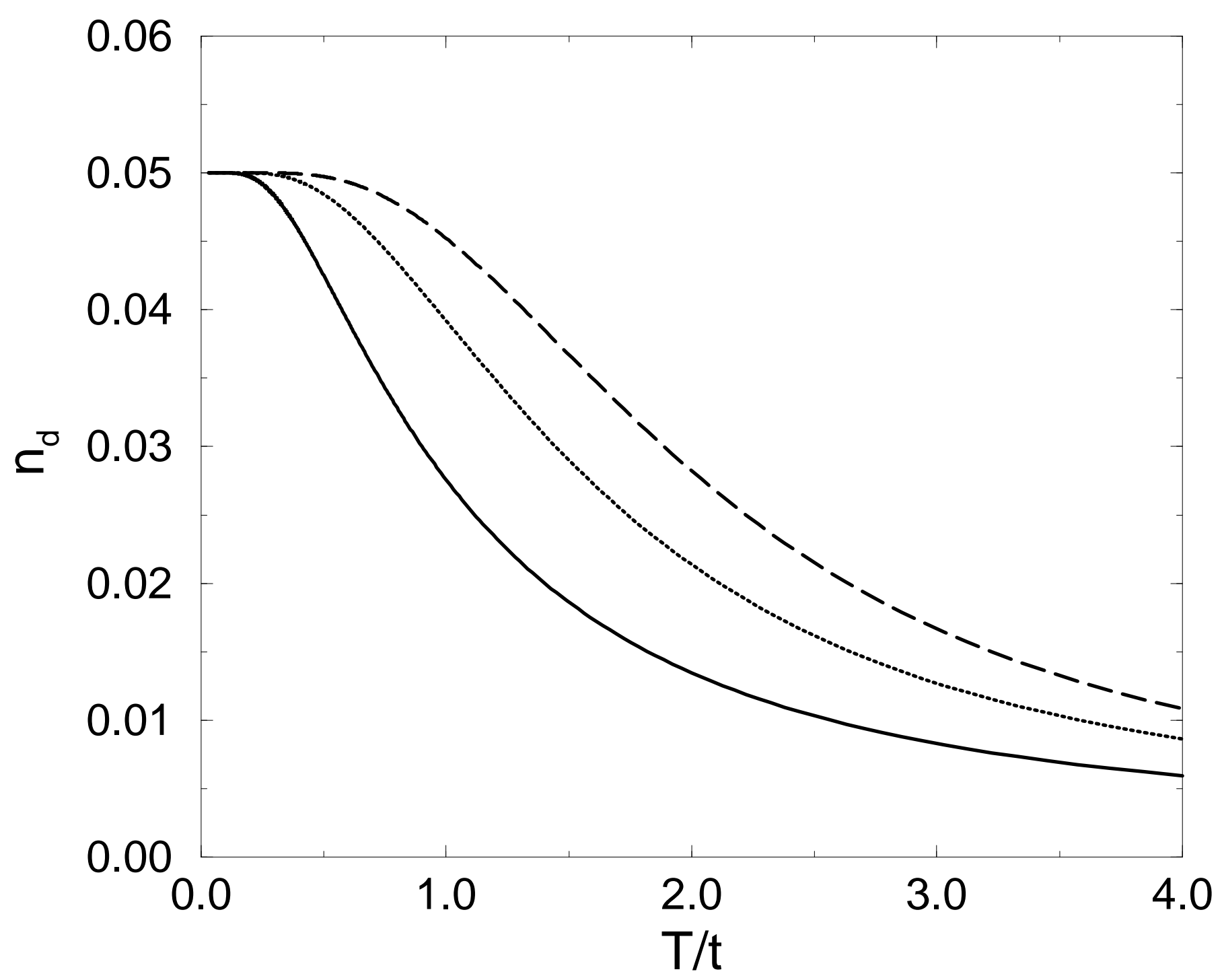

